Original paper

\title{
Factors predicting recovery of liver function after percutaneous drainage in malignant biliary obstruction: the role of hospital-acquired biliary sepsis
}

\author{
Suneed Kumar', Shakeel Masood' ${ }^{1}$, Utkarsh Srivastava ${ }^{1,2}$, Shibumon M. Madhavan', Smita Chauhann', Anshuman Pandey ${ }^{1}$ \\ 'Dr. Ram Manohar Lohia Institute of Medical Sciences, Lucknow, India \\ ${ }^{2}$ Amrita Institute of Medical Sciences, Kochi, India
}

\begin{abstract}
Aim of the study: Prolonged cholestasis adversely affects liver function. Hepatic functional recovery is mandatory prior to any surgical or medical intervention. Serum bilirubin levels correlate well with, and are a surrogate marker for, hepatocyte function. We aimed to ascertain factors responsible for slow decline of bilirubin and delayed recovery of liver function following percutaneous drainage in malignant biliary obstruction.

Material and methods: Sixty-seven patients with malignant jaundice who underwent percutaneous biliary drainage (PTBD) were followed until they achieved target bilirubin $\leqslant 3 \mathrm{mg} / \mathrm{dl}$. According to duration, patients were divided into early ( $\leqslant 6$ weeks, $n=43$ ) and late ( $>6$ weeks, $n=24$ ) groups. Various clinical, tumour-related and procedure-related factors were analysed for their contribution to delayed recovery with the $\chi^{2}$ or $t$-test. Multivariate logistic regression analysis was used to predict independent associations.

Results: Gallbladder cancer presenting with type I block was the commonest pathology. Overall demographic, clinical, tumour characteristics and procedural details were comparable between groups. Duration of jaundice $(p=0.026)$, liver involvement $(p=0.041)$, baseline total $(p=0.001)$ and direct bilirubin levels $(p<0.001)$, positive bile cultures with hospital-acquired bacteria $(p=0.031)$ were significant factors on univariate analysis. Bacterial growth was significantly greater following repeated biliary manipulations. The commonest organisms were Pseudomonas and Citrobacter spp. Number of re-instrumentations, post-procedural biliary sepsis and native biliary organisms were non-contributory. No factor was significant on multivariate analysis.

Conclusions: Factors directly linked to extent and duration of disease are validated as significant contributors to functional recovery after biliary drainage. Biliary sepsis with hospital-acquired organisms, especially following re-interventions is a significant modifiable risk-factor affecting bilirubin decline.
\end{abstract}

Key words: infection, obstructive jaundice, bilirubin, nosocomial, bile culture.

Address for correspondence:

Prof. Shakeel Masood, Dr. Ram Manohar Lohia Institute of Medical Sciences, Vibhuti Khand, Gomti Nagar, Lucknow, U.P., India, e-mail: shakeelmasood65@gmail.com

\section{Introduction}

Prolonged cholestasis secondary to malignant biliary tract obstruction causes profound changes of liver function [1]. Several animal and human studies have demonstrated the deterioration of hepatic enzyme and mitochondrial function during cholestasis, along with their gradual recovery after relieving obstruction [2]. Any intervention for the malignant disease process, with curative or palliative intent, requires the initial normalization of liver function, to prevent coagulopathy and liver dysfunction.

Serum bilirubin values closely follow liver mitochondrial function, and act as a surrogate marker of liver enzymatic action $[3,4]$. Reduction in bilirubin values following biliary drainage and relief of obstruction was found to indicate a gradual recovery of hepatic function. Various studies have put forward arbitrary 
cut-off values for serum total bilirubin for defining functional normalization, including the widely followed $\leq 3 \mathrm{mg} / \mathrm{dl}$ described by Makuuchi et al. [5]. This achieved external validation [6] and is now universally accepted as the minimum threshold value for safe intervention.

There exists a definite delay in the recovery of true enzymatic functions in comparison to biochemical values [7]. Just attaining biochemical normalcy does not necessarily indicate adequate functional return. Drainage of biliary obstruction lasting $\geq 4$ weeks takes about 6 weeks for adequate return of mitochondrial function $[8,9]$. It is therefore considered essential to wait at least 4-6 weeks following biliary drainage, to allow for complete functional recovery.

Despite adequate biliary drainage, some patients may take even longer to achieve the set target bilirubin value. This duration is crucial with regards to rapidly progressing biliary tract malignancies, and may alter the course of therapy. In this study, patients with malignant obstructive jaundice undergoing percutaneous biliary drainage were studied for rate of bilirubin reduction and time taken to achieve target bilirubin. The aim was to ascertain various factors that contributed to and predicted the rate of bilirubin recovery after biliary drainage in these patients.

The Gangetic belt of northern India has one of highest incidence rates of gallbladder cancer worldwide [10]. Despite the limited data centralization and disease notification, an age-related incidence rate (AAR) of 22/100,000 population has been estimated for this region [11]. The actual number is probably much higher. In daily practice, we encounter nearly 50 new cases of gallbladder cancer every month at our centre, with about $80 \%$ being advanced, metastatic or with jaundice [Masood S., unpublished data]. This rate differs even within India, with only onetenth the AAR in southern or western parts of the country [12]. Worldwide, regions such as Chile $(\mathrm{AAR}=$ 16-27/100,000), Japan (7/100,000), Poland (14/100,000) and southern Pakistan $(11 / 100,000)$ are also considered hot-spots for this disease [13]. Other regions such as north America, western Europe and Australia (AAR = 0.4-1.4/100,000) are low-risk geographical factors for the disease [14].

Gallbladder cancers have a female preponderance $(\mathrm{M}: \mathrm{F}=1: 2)$, with this being the commonest digestive cancer among women in northern India [15]. The highest incidence is found in the fifth to seventh decades of life [16]. Nonetheless, cases have been reported as early as the third decade. Risk factors for development of gallbladder cancer include gallstones, female gender, ethnicity, genetic susceptibility and lifestyle factors. Environmental factors such as presence of nickel, cadmium and chromium in water were found to have a positive correlation with gallbladder cancer $[12,17]$. Advanced gallbladder cancers are associated with dismal prognosis, with mean survival of 6 months, and 5-year survival rate $<5 \%$ [12].

\section{Material and methods}

\section{Study design and patients}

This was a retrospective analysis of prospectively maintained data over four years, from January 2015 to January 2019, in the Surgical Gastroenterology department of a tertiary referral hospital in Northern India. Seventy-five patients with malignant obstructive jaundice underwent percutaneous transhepatic biliary drainage (PTBD) for pre-operative or palliative intent during the study period. Of these, 8 patients were excluded and 67 patients were finally included in the study. Excluded patients were those who were lost to follow-up immediately after the procedure, or had no post-procedural biochemical investigations done. In addition, procedure-related mortality, defined as death occurring within 30 days or in the same hospital admission, were also excluded. The included patients were then followed up until April 2019. The study obtained clearance from the Institutional Ethics Committee for an observational study design.

\section{Procedure}

Patients were investigated with baseline haematological and biochemical parameters, triple-phase contrast enhanced computed tomography (CT) and magnetic resonance cholangiopancreatography (MRCP). The tumour characteristics on imaging, clinical TNM stage [18], resectability and operability status were noted. A bilirubin cut-off value of $\leq 3 \mathrm{mg} / \mathrm{dl}$ was considered as threshold for initiating chemotherapy or considering major liver resection. Four patients with distal biliary obstruction included in the study had unresectable disease, and PTBD was done with palliative intent because of failed endoscopic retrograde cholangiography (ERC) and stenting. Endoscopic naso-biliary drainage was not a commonly performed procedure in our centre and hence was not included in the study population.

For the purpose of our study, certain definitions were used as follows: intrahepatic biliary radicle dilatation (IHBRD) was graded as mild (central bilobar dilatation), moderate (dilated radicles not reaching the periphery) and gross (extensive tubular dilatation of 
peripheral radicles). Liver involvement by tumour was categorised as none, abutment, parenchymal infiltration and multiple metastases. The exact estimation of tumour burden within liver parenchyma was beyond the scope of this study.

All included patients underwent PTBD within 1-5 days of hospital admission, after correction of coagulation profile. The number of PTBDs per patient depended on the Bismuth-Corlette type [19] of block. Multiple catheters were inserted in higher level blocks with sectoral separation in the presence of an infected system and worsening biliary sepsis. Insertion was done under strict aseptic precautions by Seldinger technique [20] using a Chiba needle (Cook Medical, Bloomington, IN) under ultrasound and fluoroscopic guidance. Prophylactic single shot third generation cephalosporin antibiotic was administered prior to the procedure. In the case of repeat procedures or ongoing sepsis, amikacin was also added.

Peri-procedural complications were noted if present. In the case of a blocked catheter and poor drainage, flushing of the catheter tube with $10 \mathrm{ml}$ of sterile normal saline under aseptic precautions was performed. In the case of a persistent blocked catheter, pulled-out tube or peri-tubal leak, repeat invasive biliary instrumentation in the fluoroscopy suite was done. Patients were usually discharged from hospital when they recovered from peri-procedural discomfort and morbidity, and serum bilirubin showed a downward trend. They were called in at regular intervals for clinical follow-up and investigations. A limited subgroup of patients with good performance status, metastatic or unresectable disease, financially sound and consenting, underwent internalization with self-expanding metallic stents.

\section{Dichotomisation of data}

The duration to achieve target levels of total biliru$\mathrm{bin} \leq 3 \mathrm{mg} / \mathrm{dl}$ was noted. Duration of 6 weeks was considerate acceptable allowance for satisfactory return of liver functions, based on literature and previous experience. Based on this, the patients were stratified into two groups: group 1 (early group) in which reduction of bilirubin values was achieved $\leq 6$ weeks; group 2 (delayed group) which took longer than 6 weeks. Patients whose bilirubin values failed to return to normal were also included in the latter. The two groups were analysed for various clinical, biochemical, tumour-related, and procedure-related factors that would contribute to differences between them.

When total bilirubin values declined below $3 \mathrm{mg} / \mathrm{dl}$, patients who were initially resectable were reassessed for operability. The others who were primarily locally advanced or metastatic were referred for palliative chemotherapy, if performance status permitted.

\section{Statistical analysis and data interpretation}

Data were analysed using SPSS Statistics v.16 for Windows (SPSS Inc., Chicago, IL). Nominal data were represented as median with range, or mean $\pm \mathrm{SD}$. Categorical data were described as percentages. Comparison of categorical variables between the groups was by the Pearson $\chi^{2}$ test, the Fisher exact test being utilised for $2 \times 2$ cross-table analysis with small sample sizes.

Ordinal data were assumed for equality of variance by Levene's test, and analysed by the independent $t$-test or Mann-Whitney $U$ test. Results were presented as mean difference between the groups with standard error difference, along with the $95 \%$ confidence interval (CI). A two-tailed $p$-value of $<0.05$ was considered significant.

\section{Univariate and multivariate analysis}

Individual categorical variables were analysed with the $\chi^{2}$ test for significant differences between the groups. Ordinal data found significant were plotted with a receiver operator characteristics (ROC) curve for optimal cut-off and sensitivity. The optimal cut-off was estimated at the top-left most point on the curve, corresponding to the highest true positive (TP) and lowest false positive (FP) values. Area under curve (AUC) between 0.7 and 0.8 was considered a fair predictor of diagnostic ability, and that below 0.7 as poor predictor.

Variables found significant on univariate were analysed for independent association by multiple logistic regression. Results were expressed as $p$-value, odds ratio (OR) and 95\% CI.

\section{Survival analysis}

Overall survival was plotted as Kaplan-Meier curves for the two groups. The difference between the groups was estimated with the log rank test.

\section{Results}

\section{Overall demographics}

Among the 67 patients included in the study, 39 (58.2\%) were female. The mean age of the population was $54.4 \pm 12.09$ years. Apart from jaundice, the commonest symptomatology at presentation was anorexia 
and weight loss (94\%), pruritus (74.6\%) and abdominal pain (64.2\%). Patients were symptomatic for a median duration of 2 months (range: 7 days to 1.5 years). Median hospital stay was about 9 days (range: 1-53 days).

Table 1. Tumour characteristics in the two groups

\begin{tabular}{|c|c|c|c|}
\hline Variables & $\begin{array}{c}\text { Group } 1(n=43) \\
n(\%)\end{array}$ & $\begin{array}{c}\text { Group } 2(n=24) \\
n(\%)\end{array}$ & $P$-value \\
\hline Tumour site & & & 0.468 \\
\hline Gallbladder & $26(60.5)$ & $19(79.2)$ & \\
\hline Gallbladder fossa & $5(11.6)$ & $2(8.3)$ & \\
\hline CBD/hilum & $8(18.6)$ & $3(12.5)$ & \\
\hline Pancreas & $4(9.3)$ & $0(0)$ & \\
\hline IHBRD grade & & & 0.556 \\
\hline Mild & $4(9.3)$ & $1(4.2)$ & \\
\hline Moderate & $32(74.4)$ & $17(70.8)$ & \\
\hline Gross & $7(16.3)$ & $6(25.0)$ & \\
\hline Liver involvement & & & $0.041^{*}$ \\
\hline Normal & $16(37.2)$ & $3(12.5)$ & \\
\hline Abut & $2(4.7)$ & $2(8.3)$ & \\
\hline Infiltration & $21(48.8)$ & 19 (79.2) & \\
\hline Metastasis & $4(9.3)$ & $0(0)$ & \\
\hline EHBO type (Bismuth- & ette) & & 0.527 \\
\hline I & $24(55.8)$ & $11(45.8)$ & \\
\hline II & $10(23.2)$ & $7(29.2)$ & \\
\hline III A\&B & $7(16.3)$ & $6(25.0)$ & \\
\hline IV & $2(4.7)$ & $0(0)$ & \\
\hline TNM cT stage & & & 0.605 \\
\hline $\mathrm{T} 1$ & $2(4.7)$ & $0(0)$ & \\
\hline $\mathrm{T} 2$ & $9(20.9)$ & $7(29.2)$ & \\
\hline T3 & $23(53.5)$ & $11(45.8)$ & \\
\hline T4 & $9(20.9)$ & $6(25.0)$ & \\
\hline TNM cN stage & & & 0.935 \\
\hline NO & $5(11.6)$ & $3(12.5)$ & \\
\hline N1 & 27 (62.8) & $14(58.3)$ & \\
\hline N2 & $11(25.6)$ & $7(29.2)$ & \\
\hline TNM cM stage & & & 0.751 \\
\hline MO & $32(74.4)$ & $17(70.8)$ & \\
\hline M1 & $11(25.6)$ & $7(29.2)$ & \\
\hline AJCC $8^{\text {th }}$ stage groupi & & & 0.648 \\
\hline I & $4(9.3)$ & $1(4.2)$ & \\
\hline III A & $3(7.0)$ & $0(0)$ & \\
\hline III B & $16(37.2)$ & 10 (41.7) & \\
\hline IV A & $3(7.0)$ & $2(8.3)$ & \\
\hline IV B & $17(39.5)$ & $11(45.8)$ & \\
\hline
\end{tabular}

CBD - common bile duct, IHBRD - intrahepatic biliary radiclec dilatation, EHBO - extrahepatic biliary obstruction, TNM - tumour, node, metastasis, ACCC - American Joint Committee on Cancer ${ }^{*} p$-value $<0.05$, significant
Ultrasonography showed a detectable mass in nearly $95 \%$ of cases. Gallbladder was the commonest site of tumour in $57.2 \%$ cases, with associated gallstones present in $22.4 \%$. The commonest level of obstruction was type I Bismuth-Corlette. Eighty-two percent of patients were referred for palliative PTBD in view of locally unresectable or metastatic disease at presentation. Among the 12 patients who were initially resectable, none of them proceeded to surgery following normalisation of liver functions. Eight patients had disease progression, three patients refused surgery, and one did not turn up for restaging. Only $13.4 \%$ of the population received palliative chemotherapy after drainage, indicating poor overall disease outcomes.

Although all patients had technically successful PTBD insertion, functional success, measured by the ability to reach the target total bilirubin value of $\leq 3 \mathrm{mg} / \mathrm{dl}$, was achieved in only 46 patients $(68.6 \%)$. The median time for the study population to achieve this target value was 30 days. Thus, the chosen cut-off duration (6 weeks) for group allotment allowed more flexibility and leniency to allow patient inclusion. There were 43 patients in group 1 (early) and 24 in group 2 (late). The 21 patients who could not achieve target bilirubin until the end of the follow-up period were included in group 2.

\section{Group-wise demographics}

The overall patient demographics and tumour characteristics were comparable between the groups. Group 1 had more female subjects than males, but the distribution was not significantly different (group 1, $\mathrm{M}: \mathrm{F}=15: 28$, group $2, \mathrm{M}: \mathrm{F}=13: 11, p=0.125$ ). The mean ages were also similar, $55.11 \pm 12.96$ years and $53.79 \pm 10.53$ years, respectively $(p=0.670)$. Mean duration of symptoms was significantly different between groups: $2.72 \pm 2.86$ months in group 1 and $5.72 \pm 7.73$ months in group $2(p=0.026)$.

On routine blood investigations, most haematological and biochemical parameters were comparable between groups. Baseline values of serum total bilirubin (16.03 \pm 6.68 vs. $21.27 \pm 4.68 \mathrm{mg} / \mathrm{dl}, p=0.001)$ and direct bilirubin $(8.24 \pm 3.14$ vs. $11.36 \pm 2.68 \mathrm{mg} / \mathrm{dl}$, $p<0.001$ ) were the only significantly different factors between the groups.

\section{Tumour-related characteristics}

Gallbladder neck was the commonest site of tumour causing obstructive jaundice in both groups (group 1, $30.2 \%$, group 2, $54.4 \%$ ). Most tumours showed liver involvement with moderate to gross IHBRD (Table 1). 
Locally advanced disease with surrounding organ infiltration was seen in $16.3 \%$ in group 1 and $25.0 \%$ in group $2(p=0.387)$; duodenum was the most frequently involved extra hepato-biliary organ $(13.9 \%$ and $26.0 \%$, respectively). Extrahepatic metastases were evident in $14.0 \%$ and $16.7 \%$ ( $p=0.765)$; the commonest site was the peritoneal surface $(11.6 \%$ and $12.5 \%$, respectively).

Biliary cancers (gallbladder and hilar cholangiocarcinoma) were categorised according to TNM stages as mentioned in Table 1. Overall stage groups were statistically comparable between the two groups. Only $23.3 \%$ in group 1 and $8.3 \%$ in group 2 were clinically resectable lesions at primary presentation $(p=0.105)$. Patients were labelled inoperable in $39.5 \%$ and $29.2 \%$ of cases, and locally unresectable in $37.2 \%$ and $62.5 \%$, respectively in each group (non-significant difference).

\section{Procedure details}

Most patients underwent single PTBD; multiple PTBDs were done in approximately $32 \%$ in group 1 and $20 \%$ of cases in group 2. The indication for multi-sectoral drainage was either insufficient resolution of jaundice or unresolving cholangitis in higher block patterns (Bismuth-Corlette type II and beyond). About one-third patients in each group underwent stent internalization of PTBD. The details of procedure and various complications encountered are listed in Table 2.

Bile samples for culture were sterile in nearly $37 \%$ of patients in group 1 and $20 \%$ in group 2. Among culture-positives, the commonest native bacteria isolated in bile were Escherichia coli and Enterococcus spp. Skin contaminants such as coagulase-negative Staphylococcus aureus were excluded from positive cultures. Fourteen patients over both groups showed growth of hospital-acquired bacteria on primary or repeat cultures, the commonest of which were Pseudomonas aerugino$s a$ and Citrobacter koseri. The increased growth of hospital-acquired bacteria in bile cultures in the late group was a significant differentiating factor.

Twelve patients who presented with severe biliary sepsis had fungal cultures done. Positive cultures were found in 4 patients; Candida spp. was the commonest organism isolated ( $n=3 / 4$ cases). This was not statistically different between groups.

Nearly $80 \%$ of bile culture positives for native bacteria in both groups were present in the primary culture, done at the index PTBD procedure. There was no difference in culture-growth patterns of bacteria in primary cultures between the groups. However, subsequent cultures done during repeat biliary instru-
Table 2. Procedure-related differences between the groups

\begin{tabular}{|c|c|c|c|}
\hline Variables & $\begin{array}{c}\text { Group } 1(n=43) \\
n(\%)\end{array}$ & $\begin{array}{c}\text { Group } 2(n=24) \\
n(\%)\end{array}$ & $P$-value \\
\hline Number of PTBDs & & & 0.541 \\
\hline Single & $29(67.4)$ & $19(79.2)$ & \\
\hline Double & $10(23.3)$ & $3(12.5)$ & \\
\hline Triple & $4(9.3)$ & $2(8.3)$ & \\
\hline Drainage & & & 0.981 \\
\hline External & $27(62.8)$ & $15(62.5)$ & \\
\hline Internal & $16(37.2)$ & $9(37.5)$ & \\
\hline \multicolumn{4}{|l|}{ Complications } \\
\hline Bile leak & $3(7.0)$ & $4(16.7)$ & $0.239^{*}$ \\
\hline Haemobilia & $10(23.3)$ & $5(20.8)$ & 0.820 \\
\hline Pull-out & $12(27.9)$ & $7(29.2)$ & 0.913 \\
\hline Catheter block & $13(30.2)$ & $7(29.2)$ & 0.927 \\
\hline Infection/sepsis & $17(39.5)$ & $6(25.0)$ & 0.230 \\
\hline Re-PTBD & $15(34.9)$ & $6(25)$ & 0.403 \\
\hline \multicolumn{4}{|c|}{ Bile culture positive infections (overall) } \\
\hline Native bacteria & $22(51.2)$ & $10(41.7)$ & 0.456 \\
\hline $\begin{array}{l}\text { Hospital acquired } \\
\text { bacteria }\end{array}$ & $5(12.5)$ & $9(37.2)$ & $0.031^{*}$ \\
\hline Fungal & $4(9.3)$ & $0(0)$ & $0.288^{*}$ \\
\hline \multicolumn{4}{|c|}{ Primary bile culture growth } \\
\hline Native bacteria & $18(41.8)$ & $8(33.3)$ & 0.533 \\
\hline $\begin{array}{l}\text { Hospital acquired } \\
\text { bacteria }\end{array}$ & $2(4.6)$ & $2(8.3)$ & 0.784 \\
\hline Fungal & $1(2.3)$ & $0(0)$ & 0.686 \\
\hline \multicolumn{4}{|c|}{ Repeat bile cultures growth } \\
\hline Native bacteria & $4(9.3)$ & $2(8.3)$ & 0.238 \\
\hline $\begin{array}{l}\text { Hospital acquired } \\
\text { bacteria }\end{array}$ & $3(6.9)$ & $7(29.1)$ & $0.015^{*}$ \\
\hline Fungal & $3(6.9)$ & $0(0)$ & 0.347 \\
\hline
\end{tabular}

mentation showed significantly increased growth of hospital-acquired bacteria in the delayed group, with comparable growth of native organisms.

On univariate analysis, five variables emerged as significant contributors: duration of symptomatology, degree of liver involvement, baseline serum total and direct bilirubin values and positive bile cultures with hospital-acquired bacteria.

The three nominal variables were assessed with ROC curves for optimal cut-off and diagnostic ability. Duration of symptomatology, with an optimal cut-off of 3.25 months (sensitivity $45 \%$, specificity $82 \%$ ), had a poor AUC of 0.653 . Baseline total bilirubin values had a fair AUC of 0.742 with threshold of $18.0 \mathrm{mg} / \mathrm{dl}$ 

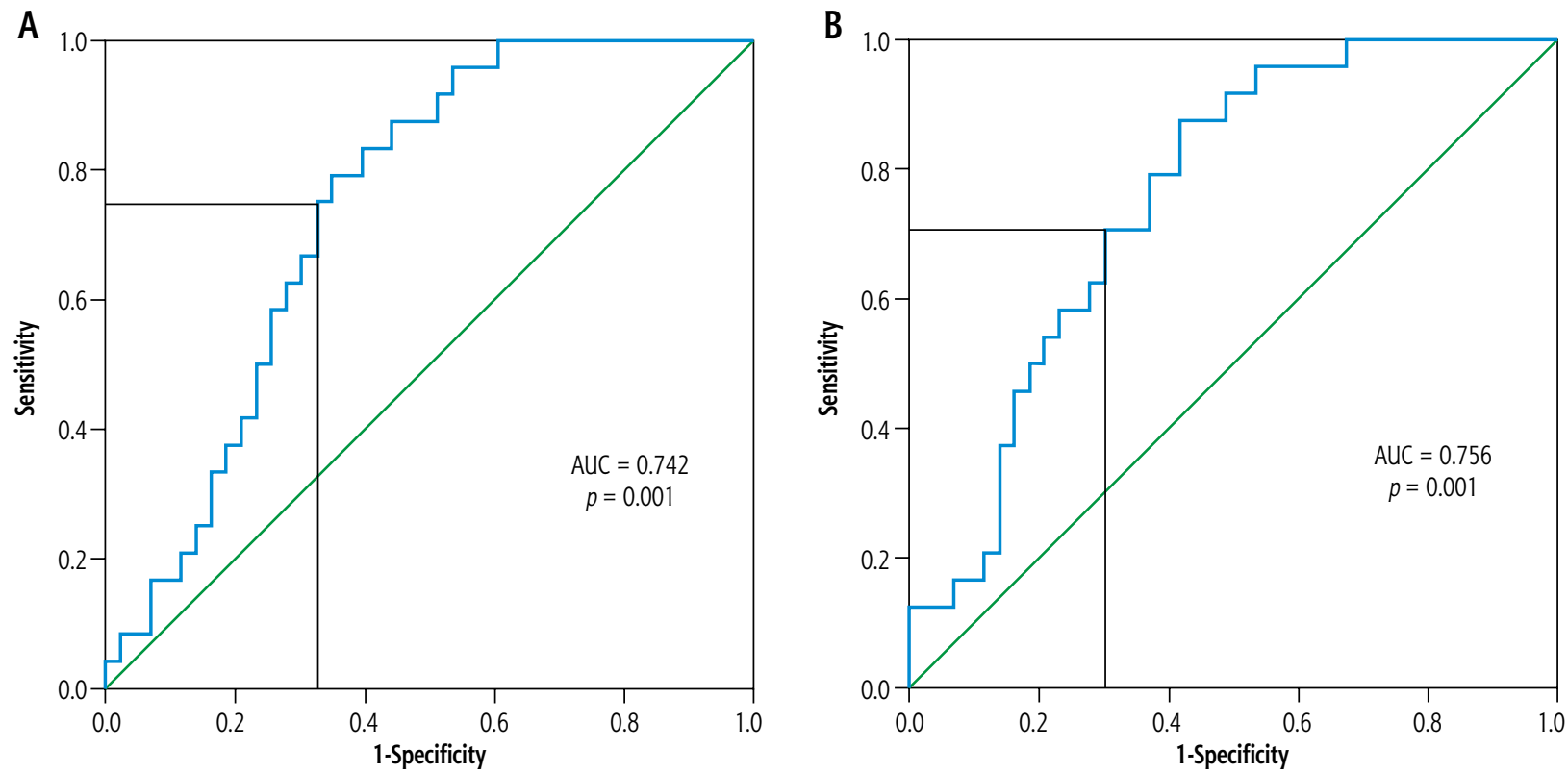

Fig. 1. A) ROC curve for baseline total bilirubin. AUC $=0.742,95 \% \mathrm{Cl}: 0.627-0.858$, Best cut-off: $18.0 \mathrm{mg} / \mathrm{dl}$, sensitivity $=79 \%$, specificity $=66 \%$, B) ROC curve for baseline direct bilirubin. AUC $=0.756,95 \% \mathrm{Cl}: 0.642-0.869$, Best cut-off: $9.26 \mathrm{mg} / \mathrm{dl}$, sensitivity $=79 \%$, specificity $=63 \%$

Table 3. Results of multivariate analysis

\begin{tabular}{lccc}
\hline Variables & $P$-value & Odds ratio & $95 \% \mathrm{Cl}$ \\
\hline Liver involvement & 0.329 & 4.023 & $0.246-65.718$ \\
\hline $\begin{array}{l}\text { Acquired bacteria positive } \\
\text { culture }\end{array}$ & 0.061 & 0.123 & $0.014-1.103$ \\
\hline Duration of symptoms & 0.093 & 0.306 & $0.077-1.217$ \\
\hline Total bilirubin & 0.999 & 0.000 & - \\
\hline Direct bilirubin & 0.999 & 0.000 & - \\
\hline
\end{tabular}

Cl - confidence interval

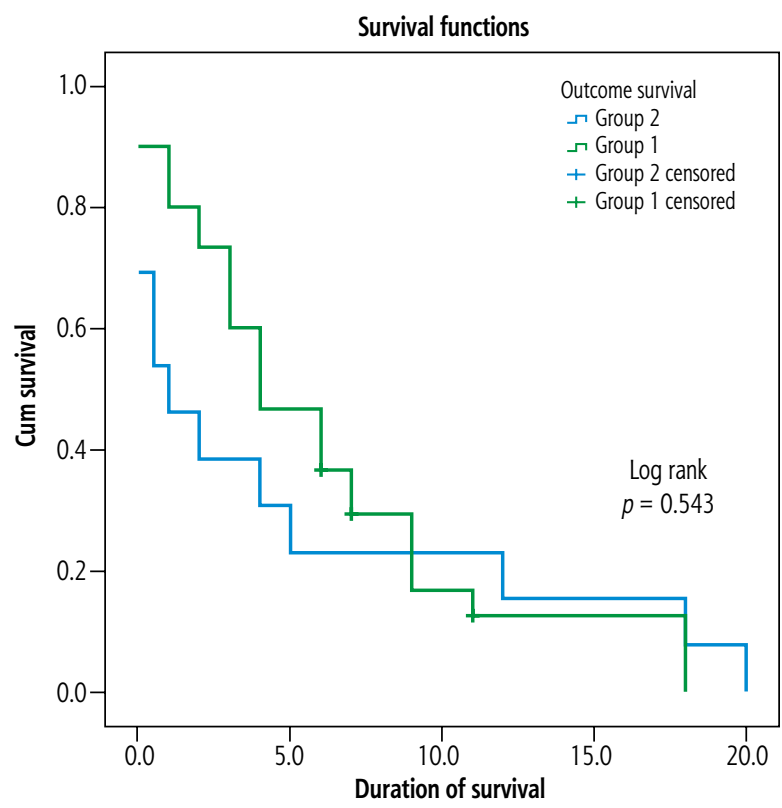

Fig. 2. Kaplan-Meier curve comparing overall survival between two groups (sensitivity 79\%, specificity 66\%) (Fig. 1A). Baseline direct bilirubin values also had a fair AUC of 0.756 with cut-off at $9.26 \mathrm{mg} / \mathrm{dl}$ (sensitivity 79\%, specificity 63\%) (Fig. 1B).

The five variables were included in multivariate analysis. No factor was found significant enough to independently contribute to the recovery rate of bilirubin after biliary drainage (Table 3 ).

\section{Survival}

The mean survival times for group 1 and group 2 were $6.14 \pm 1.0$ months and $4.84 \pm 1.97$ months respectively (Fig. 2). On log rank estimation, they were found comparable $(p=0.543)$. Hence, although faster bilirubin recovery favoured improved overall survival the difference was not statistically significant.

\section{Discussion}

Optimal recovery of liver function after drainage procedures is an often-overlooked aspect in the management of malignant biliary obstruction. Though the attainment of target values is given due importance, the actual pattern of reduction and factors modifying the rate are seldom taken into consideration. The age-old teaching states that severity and duration of biliary obstruction are the key underlying factors deciding hepatic function [21], since they determine baseline bilirubin value at presentation and degree of liver involvement. The effect of prolonged cholestasis 
on hepatocytes has been well documented by animal models $[7,8,22]$.

The recovery of liver biochemical function after biliary drainage has been studied following surgical and endoscopic drainage [23-27]. Singh et al. stated that the recovery process continues for about 6 weeks from drainage, correlating with the cut-off we chose to dichotomise our study population [9]. Pellegrini et al. demonstrated delayed recovery of bilirubin following drainage in patients with prolonged cholestasis $>4$ weeks, baseline bilirubin $>10 \mathrm{mg} / \mathrm{dl}$ and periportal fibrosis on liver biopsy [28]. The influence of underlying liver damage as evidenced on liver biopsy was also demonstrated by Negi et al. [29].

Weston et al. identified baseline bilirubin values $\geq 10 \mathrm{mg} / \mathrm{dl}$, tumour location, presence of liver metastases and baseline international normalized ratio (INR) values as factors significantly associated with rate of bilirubin decline after endoscopic drainage [26]. A similar study from China showed liver metastases to be associated with lower success rate of biliary drainage [30].

Thus, various parameters have been identified across the literature as significant contributors to biochemical recovery following biliary drainage. Among them, baseline bilirubin levels, duration of jaundice and underlying liver involvement were the factors mentioned most in different studies [25, 26, 28, 30-32]. Our study not only demonstrated the significant association of each of these factors with early bilirubin recovery; it brought forward another significant contributing variable in the form of biliary sepsis. Kato et al. demonstrated in an animal model the effect of bacterial infection in an obstructed biliary system [22]. There was impaired mitochondrial function which resulted in delayed recovery of liver function after drainage.

In our study, the growth of native organisms of the gastro-intestinal tract in bile cultures, namely Escherichia coli, Klebsiella, Enterobacter spp., etc. was not significantly different between the two groups. Despite having comparable incidence of PTBD complications (blockage, pull-out, etc.) warranting repeat biliary manipulation in both groups, the presence of culture-positive hospital-acquired organisms such as Pseudomonas spp., Citrobacter spp. and Acinetobacter spp. was significantly higher in the delayed recovery group. Biliary sepsis due to these multi-drug resistant bacteria required the administration of second-line antibiotics such as imipenem-cilastatin, colistin or piperacillin-tazobactam, based on sensitivity and institutional protocol. Multiple biliary instrumentation, repeated manipulations and prolonged hospital stay are factors which enabled entry of hospital-acquired bacteria into biliary cultures. This probably added to the insult on poorly recovering liver function and caused further deterioration.

Nearly $68 \%$ of patients with positive bile cultures for native bacteria went on to recover early. However, about $64 \%$ of patients with cultures growing hospital-acquired bacteria had delayed recovery of biochemical function. There was no difference noted between the individual organisms grown, but Pseudomonas spp. was responsible for nearly $30 \%$ of all patients within the delayed recovery group.

Clinical signs of infection and biliary sepsis after the procedure tended to be higher in the patients who recovered early. This was also associated with the higher incidence of culture positivity with native organisms in bile. However, these differences were not statistically significant. Moreover, most of the native organisms were grown in primary cultures of previously un-instrumented biliary tracts. These did not develop delayed functional recovery, probably due to the relatively healthy underlying liver with limited hepatocyte insult, and the protective effect of prophylactic antibiotics on the cholangio-venous reflux during the procedure.

On the other hand, hospital-acquired organisms, although insignificant, were seen more following repeat procedures and biliary manipulations of a previously instrumented biliary tree. Thus, the underlying cholestatic liver suffered an additional insult. Also, the spectrum of routine prophylactic antibiotics could not cover these organisms. It is probably the cumulative effect of these factors that resulted in delayed functional recovery in these patients. Although not formally studied, we have achieved some initial success using a multi-antibiotic regime and second-line drugs in the prophylaxis of repeat biliary procedures. Our antibiotic regime of choice is a combination of cefoperazone-sulbactam and amikacin; or single agent piperacillin-tazobactam. This forms a good basis for future prospective study designs.

Fungal bile cultures were not routinely performed, but only when clinically indicated. Positive cultures of Candida spp. and Trichosporon spp. were grown and required the administration of fluconazole and/or echinocandins. However, they had no contribution to delayed functional recovery. On the contrary, all the patients with biliary fungal culture positive had early recovery of bilirubin. This showed no statistical significance, and will probably need greater numbers to consider clinical significance.

None of the factors contributing significantly to delayed recovery of bilirubin were capable of independent prediction. The presence of long standing and higher levels of jaundice in a patient with significant liver involvement should be considered to indicate 
poorer recovery of liver function, in the presence of bile-culture growing hospital-acquired bacteria.

The lack of survival difference between the two groups in our study indicates the underlying disease severity and the masking of symptomatic relief of biliary drainage by the disease progression. Since $>80 \%$ of our study population was palliative, and the remaining patients also were not undergoing definitive surgery, the role of PTBD was primarily to improve the quality of life in these patients. In retrospect, whether the $8 / 12$ initially resectable patients who had disease progression during the mandatory waiting period could have been salvaged by earlier intervention, or a higher minimum bilirubin threshold, is a matter to be contemplated. Although distal biliary tumours do not require preoperative drainage, proximal tumours without hepatic functional recovery run the risk of postoperative liver failure $[33,34]$. The results of a previous retrospective analysis of a small cohort of 20 patients, who underwent major hepatectomy without any preoperative biliary drainage with acceptable outcomes [35], cannot be safely extrapolated outside a clinical trial setting.

To summarize, our study objectively validates the age-old teaching, and brings forward five significant factors that contribute to the rate of bilirubin reduction following percutaneous biliary drainage. Despite their inability to predict the outcome independently, they would give valuable insight into the natural history and behaviour of patients with malignant obstructive jaundice after biliary drainage. A novel finding is the significant contribution of hospital-acquired bacteria growth in bile culture, especially in those biliary tracts that have undergone repeated instrumentations. This seems to be a potential modifiable factor to plan future studies, directed towards targeted antibiotic prophylaxis and therapy for biliary re-interventions.

Our study is limited by the retrospective design, and the inability to include an initial prospective report of a new antibiotic regime. Confounding variables such as maintenance of strict asepsis during repeat biliary manipulation and PTBD catheter flushing may have been overlooked. Certain predictive factors could have been better elaborated, such as hepatic tumour burden, which was limited by data availability during retrospective analysis. The analysis and description of pattern of reduction of total and direct bilirubin were too exhaustive and were not included in the preparation of this manuscript.

\section{Conclusions}

The recovery of liver biochemical function following biliary drainage is influenced by the duration of block, degree of liver involvement, baseline total and direct bilirubin values. The role of biliary sepsis with growth of nosocomial organisms in bile cultures, especially after repeated biliary manipulations, shows a significant and possibly modifiable predictive factor for bilirubin recovery.

\section{Acknowledgements}

We acknowledge the contribution of Mr. Manoj Pandey (M/S Research Solutions, Lucknow) with the statistical analysis, and Mr. Pankaj Patel with the preparation of the manuscript.

\section{Disclosure}

The authors declare no conflict of interest.

\section{References}

1. van der Gaag NA, Kloek JJ, de Castro SMM, et al. Preoperative biliary drainage in patients with obstructive jaundice: History and current status. J Gastrointest Surg 2009; 13: 814-820.

2. Wang C, Xu Y, Lu X. Should preoperative biliary drainage be routinely performed for obstructive jaundice with resectable tumor? Hepatobiliary Surg Nutr 2013; 2: 266-271.

3. Aronsen KF. Liver function studies during and after complete extrahepatic biliary obstruction in the dog. Acta Chir Scand 1961; Suppl 275: 1-114.

4. Ozawa K, Takasan H, Kitamura O, et al. Alteration in liver mitochondrial metabolism in patients with biliary obstruction due to liver carcinoma. Am J Surg 1973; 126: 653-657.

5. Makuuchi M, Thai BL, Takayasu K, et al. Preoperative portal embolization to increase safety of major hepatectomy for hilar bile duct carcinoma: a preliminary report. Surgery 1990; 107: 521-527.

6. Nimura Y, Hayakawa N, Kamiya J, et al. Hepatic segmentectomy with caudate lobe resection for bile duct carcinoma of the hepatic hilus. World J Surg 1990; 14: 535-543.

7. Miyata K. Delayed recovery of mitochondrial function in rat liver after releasing biliary obstruction. Nagoya J Med Sci 1983; 45 : 97-105.

8. Kanai M, Tanaka M, Nimura Y, et al. Mitochondrial dysfunction in the non-obstructed lobe of rat liver after selective biliary obstruction. Hepatogastroenterology 1992; 39: 385-391.

9. Singh V, Kapoor VK, Saxena R, et al. Recovery of liver functions following surgical biliary decompression in obstructive jaundice. Hepatogastroenterology 1998; 45: 1075-1081.

10. Phadke PR, Mhatre SS, Budukh AM, Dikshit RP. Trends in gallbladder cancer incidence in the high- and low-risk regions of India. Indian J Med Pediatr Oncol 2019; 40: 90-93.

11. Stinton LM, Shaffer EA. Epidemiology of gallbladder disease: cholelithiasis and cancer. Gut Liver 2012; 6: 172-187.

12. ICMR Subcommittee on gallbladder cancer. Consensus document for management of gallbladder cancer 2014. New Delhi: Division of Publication and Information, Indian Council of Medical Research. Available at: https://main.icmr.nic.in/sites/ default/files/guidelines/GALLBLADDER\%20CANCER_0.pdf.

13. Randi G, Franceschi S, La Vecchia C. Gallbladder cancer worldwide: geographical distribution and risk factors. Int J Cancer 2006; 118: 1591-1602. 
14. Vijayakumar A, Vijayakumar A, Patil V, et al. Early diagnosis of gallbladder carcinoma: an algorithm approach. ISRN Radiol 2012; 2013: 239424.

15. Dhir V, Mohandas KM. Epidemiology of digestive tract cancers in India IV. Gall bladder and pancreas. Indian J Gastroenterol 1999; 18: 24-28.

16. Murthy NS, Rajaram D, Gautham MS, et al. Trends in incidence of gallbladder cancer - Indian scenario. Gastrointestinal cancer: Targets and therapy 2011; 1: 1-9.

17. Kanthan R, Senger JL, Ahmed S, Kanthan SC. Gallbladder cancer in the 21st century. J Oncol 2015; 2015: 967472.

18. Amin MB, Edge SB, Greene FL, et al. American Joint Committee on Cancer. AJCC Cancer Staging Manual. $8^{\text {th }}$ ed. Springer, New York, NY 2017.

19. Bismuth H, Corlette MB. Intrahepatic cholangioenteric anastomosis in carcinoma of the hilus of the liver. Surg Gynecol Obstet 1975; 140: 170-178.

20. Ferrucci Jr. JT, Mueller PR. Intervention radiology of the biliary tract. Gastroenterol 1982; 82: 974-985.

21. Ritchie HD. Surgical jaundice. Aspects of pathogenesis and diagnosis. Ann R Coll Surg Eng 1973; 52: 254-260.

22. Kato S, Nagano I, Nimura Y, et al. Hepatic recovery after biliary drainage in experimental obstructive jaundice complicated by biliary infection. Hepatogastroenterology 1994; 41: 217-221.

23. Nimura Y, Kamiya J, Kondo S, et al. Aggressive preoperative management and extended surgery for hilar cholangiocarcinoma: Nagoya experience. J Hepatobiliary Pancreat Surg 2000; 7: 155-162.

24. Kawasaki S, Imamura H, Kobayashi A, et al. Results of surgical resection for patients with hilar bile duct cancer: application of extended hepatectomy after biliary drainage and hemihepatic portal vein embolization. Ann Surg 2003; 238: 84-92.

25. Watanapa P. Recovery patterns of liver function after complete and partial surgical biliary decompression. Am J Surg 1996; 171 : 230-234.

26. Weston BR, Ross WA, Wolff RA, et al. Rate of bilirubin regression after stenting in malignant biliary obstruction for the initiation of chemotherapy. Cancer 2008; 112: 2417-2423.

27. Hong SK, Jang JY, Kang MJ, et al. Comparison of clinical outcome and cost effectiveness after various preoperative biliary drainage methods in periampullary cancer with obstructive jaundice. J Korean Med Sci 2012; 27: 356-362.

28. Pellegrini CA, Thomas MJ, Way LW. Bilirubin and alkaline phosphatase values before and after surgery for biliary obstruction. Am J Surg 1982; 143: 67-73.

29. Negi SS, Chaudhary A. Analysis of abnormal recovery pattern of liver function tests after surgical repair of bile duct strictures. J Gastroenterol Hepatol 2005; 20: 1533-1537.

30. Zhang GY, Li WT, Peng WJ, et al. Clinical outcomes and prediction of survival following percutaneous biliary drainage for malignant obstructive jaundice. Oncol Lett 2014; 7: 1185-1190.

31. Kawarada Y, Higashiguchi T, Yokoi H, et al. Preoperative biliary drainage in obstructive jaundice. Hepatogastroenterology 1995; 42: 300-307.

32. Langman EL, Suhocki PV, Hurwitz HI, et al. Percutaneous biliary drainage catheter insertion in patients with extensive hepatic metastatic tumor burden. J Gastrointest Oncol 2016; 7: 875-881.

33. Saxena P, Kumbhari V, Zein MEL, Khashab MA. Preoperative biliary drainage. Digest Endosc 2015; 27: 265-277.

34. Moole H, Bechtold M, Puli SR. Efficacy of preoperative biliary drainage in malignant obstructive jaundice: a meta-analysis and systematic review. World J Surg Oncol 2016; 14: 182.
35. Cherqui D, Benoist S, Malassagne B, et al. Major liver resection for carcinoma in jaundiced patients Without preoperative biliary drainage. Arch Surg 2000; 135: 302-308. 